\#DLFteach • \#DLFTeach Toolkit: Lesson Plans for Digital Library Instruction

\title{
Getting Started with Data Visualization: Tools for Research!
}

\author{
Alison Blaine, Jennifer Garrett
}

Published on: Sep 19, 2019

DOI: $10.21428 / 65 a 6243 c .1566620 a$

License: Creative Commons Attribution 4.0 International License (CC-BY 4.0). 


\section{Session Specifics}

This is a 90-minute workshop that covers the basics of creating data visualizations and provides hands-on exercises using two tools that are freely available to students:

Tableau and RAWGraphs. To shorten the workshop to 60 minutes, take out the RAWGraphs activity.

\section{Instructional Partners}

Instructors on campus wanting their students to learn the basics of data visualization.

\section{Audience}

- Undergraduates in courses that may require the production of a data visualization as part of an assignment or final project.

- Graduate students, postdocs, \& faculty with research questions, projects or presentations that require data visualization for exploratory purposes or final delivery.

- Staff trying to explore new tools for their work.

- Note: for all audience members, a basic understanding of tabular data structure (columns and rows) and file formats (be able to distinguish between Excel and CSV file types) will be helpful to have in this workshop.

\section{Curricular Context}

This workshop can be taught in any curricular context and was not designed as coursespecific.

\section{Learning Outcomes}

Following the session, participants should leave with:

1. A basic understanding of the process for making good visualizations.

2. Gain beginning proficiency with Tableau, being able to do the following actions:

- Import a dataset

- Create basic charts, including a line chart and map

- Edit map colors

- Create a drop-down filter for users to filter data

- Create a data dashboard

- Be able to change the position of charts on the dashboard 
- Make a dynamic chart title that changes based on dropdown menu selection

- Publish your Tableau workbook to your Tableau Public account

3. Gain beginning proficiency with RAWGraphs, being able to do the following actions:

- Import data

- Select a chart type

- Select fields for building the chart

- Build a small multiples chart

- Build a circle-packing chart

- Export the charts as image or vector file

\section{Preparation}

1. Laptops or computers with Tableau Public installed are required for the hands-on portion of this course. This software can be downloaded from Tableau.

2. Participants need to create a Tableau Public account in order to be able to publish their visualizations to the web. Note that if you are using Tableau Public, you cannot save your work directly to your computer. It must be saved to the online gallery.

3. Participants will need to download the dataset in the workshop materials folder for use in Tableau and RAWGraphs.

\section{Materials}

Instructor \& Audience - Workshop materials can be found in the Additional Instructional Materials section below.

\section{Session Outline}

\section{Presentation}

\section{5 minutes}

Provide an overview of the data visualization process and introduce tools for creating data visualizations. Download and open slides from Additional Instructional Materials below. Important: review speaker notes in slides for detailed steps.

\section{Hands-on data visualization activity with Tableau.}

\section{5 minutes}

See guide with step-by-step instructions in Additional Instructional Materials below. 


\section{Hands-on data visualization activity with RAWGraphs}

\section{0 minutes}

See guide with step-by-step instructions in Additional Instructional Materials below.

\section{Evaluation and free exploration time}

\section{0 minutes}

\section{Assessment}

We use the following questions to capture feedback about our workshops. We generally print this form on a half-sheet of paper or create an electronic version using a free tool, such as Google Forms. Here's what that looks like:

1. How would you rate this session overall on a scale of 1 to 10 , with 10 being the highest?

2. What was the most useful thing you learned in today's session?

3. Was the session's content appropriately geared toward your learning needs? (Yes/No)

4. Do you feel you have gained a basic understanding of what is involved in creating a good visualization?

5. Do you feel that you have attained beginning proficiency with Tableau (as defined in the learning outcomes) as a result of this workshop? Please explain.

6. Do you feel that you have attained beginning proficiency with RAWGraphs (as defined in the learning outcomes) as a result of this workshop? Please explain.

7. How might the session's content have been better?

\section{Reflection}

We found when trying to teach this as an hour-long workshop that participants wanted more time for experimentation and play with the software. Making this into a 90minute session made it easier to cover the material we wanted to cover and allowed participants to have some time to explore at the end, either using the sample data sets or their own data.

Focusing on the visualization process and leaving out data gathering and cleaning made for a more focused workshop, but some participants noted that they wanted to learn more about those areas. To meet their needs, we developed a series of workshops covering different areas. 
It was also important to select stable tools that were compatible across PC and Mac environments in both selecting tools for the hands-on activity and the additional links we chose to share.

\section{Additional Instructional Materials}

\section{Slides}

Getting Started With Data Visualization: Tools for Research!-presentation with detailed speaker notes

Handout

Activity Guide with step-by-step instructions

\section{Sample data}

Sample data in csv format 